\title{
TUNNELLING RADIATION OF CHARGED PARTICLES FROM A HOŘAVA-LIFSHITZ GRAVITY BLACK HOLE
}

\author{
G.-Q. Li, Y.-Y. Ou, and Z.-T. Lin \\ Institute of Theoretical Physics, Lingnan Normal University, Zhanjiang, 524048, Guangdong, China \\ Email: ligq@lingnan.edu.cn
}

Received 27 May 2018; revised 30 June 2018; accepted 15 October 2018

\begin{abstract}
The Hawking radiation of charged particles from black holes in the Hořava-Lifshitz (HL) gravity is investigated by using the Parikh-Wilczek (PW) method, and the emission rate is calculated. The emission spectrum is not purely thermal and is consistent with an underlying unitary theory. Some other characteristics exist for a HL gravity black hole. Assuming the conventional tunnelling rate associated with the change of entropy, the entropy of the HL gravity black hole is obtained. The entropy is not proportional to the horizon area because a logarithmic term exists. However, it complies with the first law of thermodynamics and is in accord with earlier results.
\end{abstract}

Keywords: tunnelling radiation, Hořava-Lifshitz gravity, first law of thermodynamics, black hole PACS: 04.70.Dy

Hawking proved that a black hole can radiate thermally [1]. To prove that the energy spectrum is precisely thermal, much work has been done [2-9]. From a precisely thermal spectrum, we can acquire one temperature parameter but not any other information. That is, if things are absorbed into a black hole, their important information will be lost during the emission. In 2005, Hawking changed his view and thought that information is preserved and can indeed get out of the black hole [10. The change may be related to the work of Parikh and Wilczek [11-13]. By treating the Hawking radiation as a tunnelling process, Parikh and Wilczek successfully calculated the modified emission spectrum of the spherically symmetric black holes. Later, the Parikh-Wilczek (PW) method was used to study the Hawking radiation of particles from other spherically symmetric black holes [14-19] and the method was also extended to calculate the emission spectrum of other symmetric black holes [20-29]. But, they all are Einstein gravity black holes. Recently, we extended this method to modified gravity black holes [30-32] and found some significant and interesting phenomena. In this paper, we discuss the black holes in the Hořava-Lifshitz (HL) gravity and investigate the modified emission spectrum of charged particles from their event horizons. This extension is of interest because the HL gravity, proposed by Hořava, is a nonrelativistic renormalizable theory of gravity at a Lifshitz point [33]. It provides such a fascinating framework that one can explore the relation between the ordinary gravity and the string theory. For all we know, the tunnelling radiation from a HL gravity black hole has not been covered in the literature yet.

For simplicity, the dynamical coupling constant $\lambda$ in the HL theory, which is susceptible to the quantum correction [31], can be set to one. Then the metric of a spherical black hole is given as [34, 35] 


$$
\begin{aligned}
& \mathrm{d} s^{2}=-N_{0} f(r) \mathrm{d} t^{2}+\frac{\mathrm{d} r^{2}}{f(r)}+r^{2}\left(\mathrm{~d} \theta^{2}+\sin ^{2} \theta \mathrm{d} \varphi^{2}\right), \\
& f(r)=1+x^{2}-\sqrt{c_{0} x-\frac{q^{2}}{2}}, x=\sqrt{-\Lambda} r
\end{aligned}
$$

where $\Lambda$ is the negative cosmological constant, $c_{0}$ and $q$ are integration constants, and $N_{0}$ can be set to one by adjusting the time coordinate $t$.

The event horizon radius $r_{\mathrm{H}}$ is the largest positive root of the equation $f(r)=0$ and can be used to denote the thermodynamic quantities of the black hole. The Hawking temperature of the black hole is

$$
T_{\mathrm{H}}=\frac{f^{\prime}\left(r_{\mathrm{H}}\right)}{4 \pi}=\frac{6 x_{+}^{4}+4 x_{+}^{2}-2-q^{2}}{16 \pi x_{+}\left(x_{+}^{2}+1\right)} \sqrt{-\Lambda},
$$

where $\quad x_{+}=\sqrt{-\Lambda} r_{\mathrm{H}}$ and $f^{\prime}(r)=\frac{\mathrm{d} f(r)}{\mathrm{d} r}$. Because $\frac{\mathrm{d} T_{\mathrm{H}}}{\mathrm{d} x_{+}}=\frac{\sqrt{-\Lambda}}{8 \pi}\left(3+\frac{1}{x_{+}^{2}}\right) \geq 0$, the temperature always increases when $x_{+}$grows. Obviously, there exists an extremal limit for a neutral black hole, $x_{+}=1 / \sqrt{3}$, where the temperature becomes zero.

In units of $c=G=1$, the mass, electric charge and electric potential of the black hole at the horizon can be rewritten as [34]

$$
M=\frac{c_{0}}{4 \sqrt{-\Lambda}}, Q=\frac{q}{4 \sqrt{-\Lambda}}, \phi=\frac{q}{x_{+}},
$$

where

$$
c_{0}=\frac{2\left(x_{+}^{2}+1\right)^{2}+q^{2}}{2 x_{+}} .
$$

To apply the PW method, we make a transformation $\mathrm{d} T=\mathrm{d} t+\mathrm{g}(r) \mathrm{d} r$, where $g(r)$ is a function of $r$. When $f(r)+f^{2}(r) g^{2}(r)=1$ is satisfied, Eq. (1) changes into the Painlevé line element, i.e.

$$
\begin{aligned}
& \mathrm{d} s^{2}=-f(r) \mathrm{d} T^{2}+2 \sqrt{1-f(r)} \mathrm{d} T \mathrm{~d} r+\mathrm{d} r^{2} \\
& +r^{2}\left(\mathrm{~d} \theta^{2}+\sin ^{2} \theta \mathrm{d} \varphi^{2}\right) .
\end{aligned}
$$

It is obvious that the metric (6) shows us a stationary and nonsingular spacetime and it is the flat Euclidean space in radial. The Painlevé line element (6) also satisfies the Landau's condition of coordinate clock synchronization which is given by 36 ]

$$
\frac{\partial}{\partial x^{j}}\left(-\frac{g_{0 i}}{g_{00}}\right)=\frac{\partial}{\partial x^{i}}\left(-\frac{g_{0 j}}{g_{00}}\right), i, j=1,2,3 .
$$

This characteristic is indispensable for us to discuss the particle's tunnelling because it is an instantaneous quantum mechanical process.

The equation of motion of an outgoing massless particle is

$$
\dot{r}=\frac{\mathrm{d} r}{\mathrm{~d} T}=1-\sqrt{1-f(r)} .
$$

The geodesic (8) is radial-lightlike and not suitable for massive quanta whose world-line is timelike. Akin to Refs. [14, 15], when a massive particle tunnels across the horizon, we can treat it as a de Broglie wave and get its equation of motion

$$
\dot{r}=-\frac{g_{00}}{g_{01}}=\frac{f(r)}{2 \sqrt{1-f(r)}} .
$$

Since we aim to investigate the tunnelling of charged particles from a charged black hole, the effect of the electromagnetic field outside the black hole should be considered. On the one hand, we take energy and charge conservation into account and think of the outgoing charged particle as an equivalent electric charge and a de Broglie wave. Therefore, when a particle of energy $\omega$ and charge $\widetilde{Q}$ tunnels out of the event horizon, the mass and charge of the black hole will become $M-\omega$ and $Q-\widetilde{Q}$ and all the above relevant equations must be used with the replacement $M \rightarrow M-\omega$ and $Q \rightarrow Q-\widetilde{Q}$. On the other hand, we consider the black hole and the electromagnetic field as a matter-gravity system. Its Lagrangian function is $L=L_{M}+L_{Q}$, where $L_{Q}=-(1 / 4) F_{\mu \nu} F^{\mu \nu}$ is the Lagrangian function of the electromagnetic field corresponding to the generalized coordinates $A_{\mu}=(\phi, 0,0,0)$. Obviously, $A_{\mu}$ does not exist in $L_{Q}$ and then $\phi$ is a cyclic coordinate. In order to eliminate the freedom corresponding to $\phi$, the imaginary part of the action could be written as [14, 25,

$$
\begin{aligned}
& \operatorname{Im} Z=\operatorname{Im} \int_{T_{\mathrm{i}}}^{T_{\mathrm{f}}}\left(L-p_{\phi} \dot{\phi}\right) \mathrm{d} T \\
& =\operatorname{Im} \int_{r_{\mathrm{i}}}^{r_{\mathrm{f}}} \int_{(0,0)}^{\left(p_{r}, p_{\phi}\right)}\left(\mathrm{d} p_{r}-\frac{\dot{\phi}}{\dot{r}} \mathrm{~d} p_{\phi}\right) \mathrm{d} r,
\end{aligned}
$$

where $p_{r}$ and $p_{\phi}$ are the canonical momenta conjugate to the coordinates $r$ and $\phi$, respectively, and $r_{i}$ and $r_{f}$ are the horizon radiuses of the black hole before and after a charged particle tunnels out. 
We take the Hamilton equation into account and have

$$
\dot{r}=\left.\frac{\mathrm{d} H}{\mathrm{~d} p_{r}}\right|_{\left(r ; \phi, p_{\phi}\right)}, \dot{\phi}=\left.\frac{\mathrm{d} H}{\mathrm{~d} p_{\phi}}\right|_{\left(\phi ; r, p_{r}\right)},
$$

where $\left.\mathrm{d} H\right|_{\left(r ; \phi, p_{\phi}\right)}=\mathrm{d} M,\left.\mathrm{~d} H\right|_{\left(\phi ; r, p_{r}\right)}=\phi \mathrm{d} Q$. Substituting Eq. (11) for (10) and switching the order of integration, we have

$$
\begin{aligned}
& \operatorname{Im} Z=\operatorname{Im}\left[\int_{\left(M_{\mathrm{i}}, Q_{\mathrm{i}}\right)}^{\left(M_{\mathrm{f}}, Q_{\mathrm{f}}\right)} \int_{r_{\mathrm{i}}}^{r_{\mathrm{f}}} \frac{1}{\dot{r}} \mathrm{~d} r \mathrm{~d} M\right. \\
& \left.-\int_{\left(M_{\mathrm{i}}, Q_{\mathrm{i}}\right)}^{\left(M_{\mathrm{f}}, Q_{\mathrm{f}}\right)} \int_{r_{\mathrm{i}}}^{r_{\mathrm{f}}} \frac{\phi}{\dot{r}} \mathrm{~d} r \mathrm{~d} Q\right] \\
& =\left\{\begin{array}{l}
\operatorname{Im}\left[\int_{\left(M_{\mathrm{i}}, Q_{\mathrm{i}}\right)}^{\left(M_{\mathrm{f}}, Q_{\mathrm{f}}\right)} \int_{r_{\mathrm{i}}}^{r_{\mathrm{f}}} \frac{1+\sqrt{1-f(r)}}{f(r)} \mathrm{d} r \mathrm{~d} M\right. \\
\operatorname{Im}\left[\int_{\left(M_{\mathrm{i}}, Q_{\mathrm{i}}\right)}^{\left(M_{\mathrm{f}}, Q_{\mathrm{f}}\right)} \int_{r_{\mathrm{i}}}^{r_{\mathrm{f}}} \frac{2 \sqrt{1-f(r)}}{f(r)} \mathrm{d} r \mathrm{~d} M\right.
\end{array}\right. \\
& \left.-\int_{\left(M_{\mathrm{i}}, Q_{\mathrm{i}}\right)}^{\left(M_{\mathrm{f}}, Q_{\mathrm{f}}\right)} \int_{r_{\mathrm{i}}}^{r_{\mathrm{f}}} \frac{1+\sqrt{1-f(r)}}{f(r)} \phi \mathrm{d} r \mathrm{~d} Q\right] \text { (massless particle) } \\
& \left.-\int_{\left(M_{\mathrm{i}}, Q_{\mathrm{i}}\right)}^{\left(M_{\mathrm{f}}, Q_{\mathrm{f}}\right)} \int_{r_{\mathrm{i}}}^{r_{\mathrm{f}}} \frac{2 \sqrt{1-f(r)}}{f(r)} \phi \mathrm{d} r \mathrm{~d} Q\right] \text { (massive particle) } \\
& =-2 \pi \int_{\left(M_{\mathrm{i}}, Q_{\mathrm{i}}\right)}^{\left(M_{\mathrm{f}}, Q_{\mathrm{f}}\right)} \frac{1}{f^{\prime}\left(r_{\mathrm{H}}\right)}(\mathrm{d} M-\phi \mathrm{d} Q),
\end{aligned}
$$

where $M_{\mathrm{i}}=M, M_{\mathrm{f}}=M-\omega ; Q_{\mathrm{i}}=Q, Q_{\mathrm{f}}=Q-\widetilde{Q}$, and we have completed $r$ integral by deforming the contour around the single pole $r=r_{\mathrm{H}}$.

From Eqs. (4) and (5) we obtain

$$
\begin{aligned}
\mathrm{d} M & =\frac{1}{4 \sqrt{-\Lambda}}\left(\frac{6 x_{+}^{4}+4 x_{+}^{2}-2-q^{2}}{2 x_{+}^{2}} \mathrm{~d} x_{+}+\phi \mathrm{d} q\right), \\
\mathrm{d} Q & =\frac{1}{4 \sqrt{-\Lambda}} \mathrm{d} q .
\end{aligned}
$$

Substituting Eqs. (3) and (13) for Eq. (12) yields

$$
\begin{aligned}
& \operatorname{Im} Z=\frac{\pi}{\Lambda} \int_{x_{\mathrm{i}}}^{x_{\mathrm{f}}}\left(x_{+}+\frac{1}{x_{+}}\right) \mathrm{d} x_{+} \\
& =\frac{\pi}{-\Lambda}\left[\left(\frac{x_{\mathrm{i}}^{2}}{2}+\ln x_{\mathrm{i}}\right)-\left(\frac{x_{\mathrm{f}}^{2}}{2}+\ln x_{\mathrm{f}}\right)\right] .
\end{aligned}
$$

In the Wentzel-Kramers-Brillouin approximation, the tunnelling probability is related to the imaginary part of the action by [37]

$$
\Gamma \sim \exp (-2 \operatorname{Im} Z) .
$$

Therefore,

$$
\Gamma \sim \exp \left[\frac{\pi}{-\Lambda}\left(x_{\mathrm{f}}^{2}+2 \ln x_{\mathrm{f}}\right)-\frac{\pi}{-\Lambda}\left(x_{\mathrm{i}}^{2}+2 \ln x_{\mathrm{i}}\right)\right] .
$$

The emission spectrum (16) is not pure thermal, but it accords with an underlying unitary theory. Moreover, we note that the emission spectrums of massive particles have the same expressions as those of massless particles.

According to the conventional tunnelling rate, which is associated with the increment of black hole entropy and was shown in all of the early references about tunnelling radiation, we have

$$
\Gamma \sim \mathrm{e}^{\Delta S},
$$

where $\Delta S=S(M-\omega, Q-\widetilde{Q})-S(M, Q)$ is the increment of the entropy before and after the emission. Combining Eqs. (16) and (17), we can obtain the entropy of a black hole in the HL gravity as

$$
S=\frac{\pi}{-\Lambda}\left(x_{+}^{2}+2 \ln x_{+}\right)+S_{0},
$$

where $S_{0}$ is a constant. The result is in agreement with that given in Refs. [34, 35].

We can rewrite Eq. (18) as

$$
S=\frac{A}{4}-\frac{\pi}{\Lambda} \ln \frac{A}{A_{0}},
$$

where $A=4 \pi r_{\mathrm{H}}^{2}$ is the horizon area of the black hole and $A_{0}=-\frac{4 \pi}{\Lambda} \mathrm{e}^{\Lambda S_{0} / \pi}$. It is obvious that the area law of black hole entropy is not satisfied. In the entropy (19), the first term is exactly one quarter of the horizon area; the second is a logarithmic term, which was considered to often appear in the quantum correction of the black hole entropy in higher derivative gravity theories [34].

Expanding the action $S(M-\omega, Q-\widetilde{Q})$ in $\omega$ and $\widetilde{Q}$, ignoring the quadratic and high-order terms of $\omega$ and $\widetilde{Q}$, we have

$$
\Delta S=\frac{16 \pi x_{+}\left(x_{+}^{2}+1\right)\left(\omega-\omega_{0}\right)}{\sqrt{-\Lambda}\left(6 x_{+}^{4}+4 x_{+}^{2}-2-q^{2}\right)}=-\beta\left(\omega-\omega_{0}\right),
$$

and then

$$
\Gamma \sim \mathrm{e}^{-\beta\left(\omega-\omega_{0}\right)},
$$


where $\omega_{0}=q \widetilde{Q} / x_{+}$, and $\beta=1 / T_{\mathrm{H}}$ is the inverse of the Hawking temperature. That is, the leading-order term in the corrected spectrum (16) gives the familiar thermal Boltzmann factor $\mathrm{e}^{-\beta\left(\omega-\omega_{0}\right)}$.

On the other hand, it is not difficult to prove that the first law of thermodynamics can well be satisfied, that is,

$$
\mathrm{d} M=T \mathrm{~d} S+\phi \mathrm{d} Q
$$

Equation (22) is a combination of the energy conservation law $\mathrm{d} M=\mathrm{d} Q_{\mathrm{h}}+\phi \mathrm{d} Q$, where $Q_{\mathrm{h}}$ is heat quantity and no other force but electric field does work, and the second law of thermodynamics $\mathrm{d} S=\mathrm{d} Q_{h} / T$. The energy conservation can be used in all processes, but the second law of thermodynamics is just effective for a reversible process. That is, the emission process has been thought as a reversible one in PW tunnelling framework and therefore the result is in accordance with the unitary theory and information is conserved.

In summary, we extend the PW method to investigate the Hawking radiation of charged particles across the event horizons of black holes in the HL gravity and to gain the emission spectra which deviate from the pure thermal spectrum but are in accord with an underlying unitary theory. The emission rate is associated with the increment of the black hole entropy and its expression in the HL gravity is the same as that in the Einstein gravity, but the area formula of black hole entropy breaks down in the HL gravity. The deviation of the emission spectrum from the purely thermal one implies that the black hole is actually a 'grey-body' and other information in addition to the temperature can be obtained from the spectrum.

\section{Acknowledgements}

The research is supported by the 'Climbing Program' Special Funds of Guangdong (Grant Nos. pdjh2018b0310 and pdjh2019b0298). It is also supported by the Natural Science Foundation and the Education Department of Guangdong Province (Grant Nos. 2016A030307051 and 2017KZDXM056).

\section{References}

[1] S.W. Hawking, Particle creation by black holes, Commun. Math. Phys. 43, 199 (1975).

[2] G.W. Gibbons and S.W. Hawking, Action integrals and partition functions in quantum gravity, Phys. Rev. D 15, 2752 (1977).

[3] S.Q. Wu and X. Cai, Hawking radiation of Weyl neutrinos in a rectilinearly non-uniformly accelerating Kinnersley black hole, Chin. Phys. 11, 661 (2002).

[4] S.Q. Wu and X. Cai, Quantum thermal effect of Dirac particles in a non-uniformly rectilinearly accelerating Kinnersley black hole, Chin. Phys. Lett. 19, 141 (2002).

[5] S.Q. Wu and X. Cai, Quantum thermal effect of Dirac particles in a nonuniformly rectilinearly accelerating black hole with electric charge, magnetic charge, and cosmological constant, Int. J. Theor. Phys. 41, 641 (2002).

[6] S.Q. Wu and X. Cai, Hawking radiation of Dirac particles in an arbitrarily accelerating Kinnersley black hole, Gen. Rel. Grav. 34, 1207 (2002).

[7] S.Q. Wu and M.L. Yan, Hawking radiation of an arbitrarily accelerating Kinnersley black hole: spin-acceleration coupling effect, Chin. Phys. Lett. 20, 1913 (2003).

[8] J.Y. Zhang and Z. Zhao, Hawking radiation of Dirac particles in a nonuniformly rectilinearly accelerating black hole with electric and magnetic charge, Acta Phys. Sin. 52, 2096 (2003) (in Chinese).

[9] B. Yang, Hawking radiation of Dirac particles in a rectilinearly accelerating Kinnersley black hole, Acta Phys. Sin. 57, 1278 (2008) (in Chinese).

[10]S.W. Hawking, Information loss in black holes, Phys. Rev. D 72, 084013 (2005).

[11]P. Kraus and F. Wilczek, Self-interaction correction to black hole radiance, Nucl. Phys. B 433, 403 (1995).

[12]M.K. Parikh and F. Wilczek, Hawking radiation as tunneling, Phys. Rev. Lett. 85, 5042 (2000).

[13]M.K. Parikh, A secret tunnel through the horizon, Int. J. Mod. Phys. D 13, 351 (2004).

[14]J.Y. Zhang and Z. Zhao, Hawking radiation of charged particles via tunneling from the 
Reissner-Nordström black hole, J. High Energy Phys. 10, 55 (2005).

[15]J.Y. Zhang and Z. Zhao, Massive particles' black hole tunneling and de Sitter tunneling, Nucl. Phys. B 725, 173 (2005).

[16]Q.Q. Jiang, S.Z. Yang, and S.Q. Wu, Hawking radiation as tunnelling from arbitrarily dimensional Reissner-Nordström de Sitter black hole, Chin. Phys. 15, 1425 (2006).

[17]D.Y. Chen, Q.Q. Jiang, H.L. Li, and S.Z. Yang, The quantum tunnelling radiation of Schwarzschild de Sitter black hole with a global monopole, Int. J. Theor. Phys. 15, 2523 (2006).

[18]Y.W. Han and S.Z. Yang, Quantum tunnelling and Hawking radiation of Schwarzschild-antide Sitter black hole with topological defect, Chin. Phys. Lett. 22, 2769 (2005).

[19]J. Ren, Z. Zhao, and C.J. Gao, Hawking radiation via tunnelling from arbitrarily dimensional Schwarzschild black holes, Chin. Phys. Lett. 22, 2489 (2005).

[20]J.Y. Zhang and Z. Zhao, New coordinates for Kerr-Newman black hole radiation, Phys. Lett. B 618, 14 (2005).

[21]J.Y. Zhang and Z. Zhao, Hawking radiation via tunneling from Kerr black holes, Mod. Phys. Lett. A 20, 1673 (2005).

[22]Q.Q. Jiang, S.Z. Yang, and H.L. Li, Quantum radiation of non-stationary Kerr-Newman-de Sitter black hole, Chin. Phys. 14, 1736 (2005).

[23]S.Z. Yang, Kerr-Newman-Kasuya black hole tunnelling radiation, Chin. Phys. Lett. 22, 2492 (2005).

[24]G.Q. Li, Hawking radiation via tunneling from Born-Infeld AdS black hole, Can. J. Phys. 94, 1369 (2016).

[25]J.Y. Zhang and J.H. Fan, Tunnelling effect of charged and magnetized particles from the Kerr-
Newman-Kasuya black hole, Phys. Lett. B 648, 133 (2007).

[26]S.Z. Yang and D.Y. Chen, Tunnelling effect of the non-stationary Kerr black hole, Chin. Phys. B 17, 817 (2008).

[27]G.Q. Li, Black plane's tunneling radiation, Europhys. Lett. 75, 216 (2006).

[28]G.Q. Li, Black string's tunnelling radiation, J. Phys. A 39, 11889 (2006).

[29]G.Q. Li, Tunneling radiation from toroidal black hole, Mod. Phys. Lett. A 22, 209 (2007).

[30]G.Q. Li and J.X. Mo, Hawking radiation via tunneling from a $d$-dimensional black hole in Gauss-Bonnet gravity, Gen. Relativ. Gravit. 49, 57 (2017).

[31]G.Q. Li, Hawking radiation and entropy of a black hole in Lovelock-Born-Infeld gravity from the quantum tunneling approach, Chin. Phys. C 41, 045103 (2017).

[32] G.Q. Li and J.X. Mo, Tunneling radiation as new perspective of understanding the thermodynamics in $f(R)$ gravity, Astrophys. Space Sci. 361, 251 (2016).

[33]P. Hořava, Quantum gravity at a Lifshitz point, Phys. Rev. D 79, 084008 (2009).

[34]R.G. Cai, L.M. Cao, and N. Ohta, Topological black holes in Hořava-Lifshitz gravity, Phys. Rev. D 80, 24003 (2009).

[35]Q.J. Cao, Y.X. Chen, and K.N. Shao, Black hole phase transitions in Hořava-Lifshitz gravity, Phys. Rev. D 83, 064015 (2011).

[36]L.D. Landau and E.M. Lifshitz, The Classical Theory of Field (Pergamon Press, London, 1975).

[37]E. Keski-Vakkuri and P. Kraus, Microcanonical D-branes and back reaction, Nucl. Phys. B 491, 249 (1997).

\title{
ELEKTRINGUৃJŲ DALELIŲ TUNELINĖ SPINDULIUOTĖ IŠ HORŽAVOS-LIFŠICO GRAVITACIJOS JUODOSIOS BEDUGNĖS
}

\author{
G.-Q. Li, Y.-Y. Ou, Z.-T. Lin
}

Lingnano normalinio universiteto Teorinès fizikos institutas, Kinija 